\title{
DREIDIMENSIONALES FE-MODELL EINES ZAHNLOSEN, MIT IMPLANTATEN VERSORGEN UNTERKIEFERS
}

\author{
Merz B., Mericske-Stern R.*, Lengsfeld M.+, Schmitt J.+ \\ Institut Straumann AG, CH-4437 Waldenburg, Schweiz \\ * Klinik für Zahnärztliche Prothetik, Universität Bern, CH-3010 Bern, Schweiz \\ + Klinik für Orthopädie, Philipps-Universität Marburg, D-35033 Marburg, Deutschland
}

\section{EINLEITUNG:}

Dentale Implantate nehmen heute eine wichtige Position ein in der Versorgung von teilweise oder ganz zahnlosen Patienten. Im Fall von Patienten mit zahnlosem Unterkiefer wird die mastikatorische und phonetische Funktion gegenüber konventionellen Prothesen schon durch eine von nur zwei Implantaten getragene Hybridprothese wesentlich verbessert, eine weitere Atrophie des proximalen Kieferkammes kann vermieden und die Sicherheit des Patienten im sozialen Umgang vergrössert werden.

Die Belastung solcher Implantate und des umliegenden Knochens war schon Gegenstand zahlreicher klinischer Untersuchungen und theoretischer Modelle. Die meisten publizierten Modelle weisen allerdings starke Vereinfachungen auf, sowohl bezüglich Geometrie als auch Materialkennwerte. Auch bezüglich der äusseren Belastungen auf die Implantate wurden bisher starke Vereinfachungen vorgenommen, indem z.B. nur die vertikale Komponente der resultierenden Belastung abgeschätzt wurde. Neuere Untersuchungen zeigen eindeutig, dass die Belastungen in der frontalen und horizontalen Ebene nicht vernachlässigt werden dürfen [1].

Es war das Ziel dieser Studie, durch den Einsatz eines CT-basierten Finite Elemente (FE) Modelles und durch dreidimensionale Kraftmessung auf Implantaten in vivo ein realistisches Modell eines zahnlosen, mit zwei Implantaten versorgten Unterkiefers zu erstellen.

\section{METHODE}

Bei 5 komplett unbezahnten Testpersonen mit einer von zwei ITI-Implantaten (Institut Straumann AG, CH-4437 Waldenburg) getragenen Hybridprothese im Unterkiefer wurden spezielle Implantat-Suprastrukturen mit integrierten, dreidimensional messenden Piezo-Transducern (Kistler AG, CH-8400 Winterthur) zum Einsatz gebracht (Fig. 1). Sämtliche Testpersonen waren schon seit über 5 Jahren mit den Implantaten versorgt. Mit Hilfe eines Plotters wurden die $2 \times 3 \mathrm{Kraftvektoren}$ für beide Implantate (vertikale Achse, latero-mediale Achse und anteroposteriore Achse) bei verschiedenen Kauaktivitäten simultan aufgezeichnet. Gemessen wurde dabei maximale zentrische Okklusion, maximales, unilaterales Beissen auf einen Kraftaufnehmer (bite plate), das Kauen von Testnahrung (hartes Brot) sowie leichtes Mahlen und Zähneknirschen.

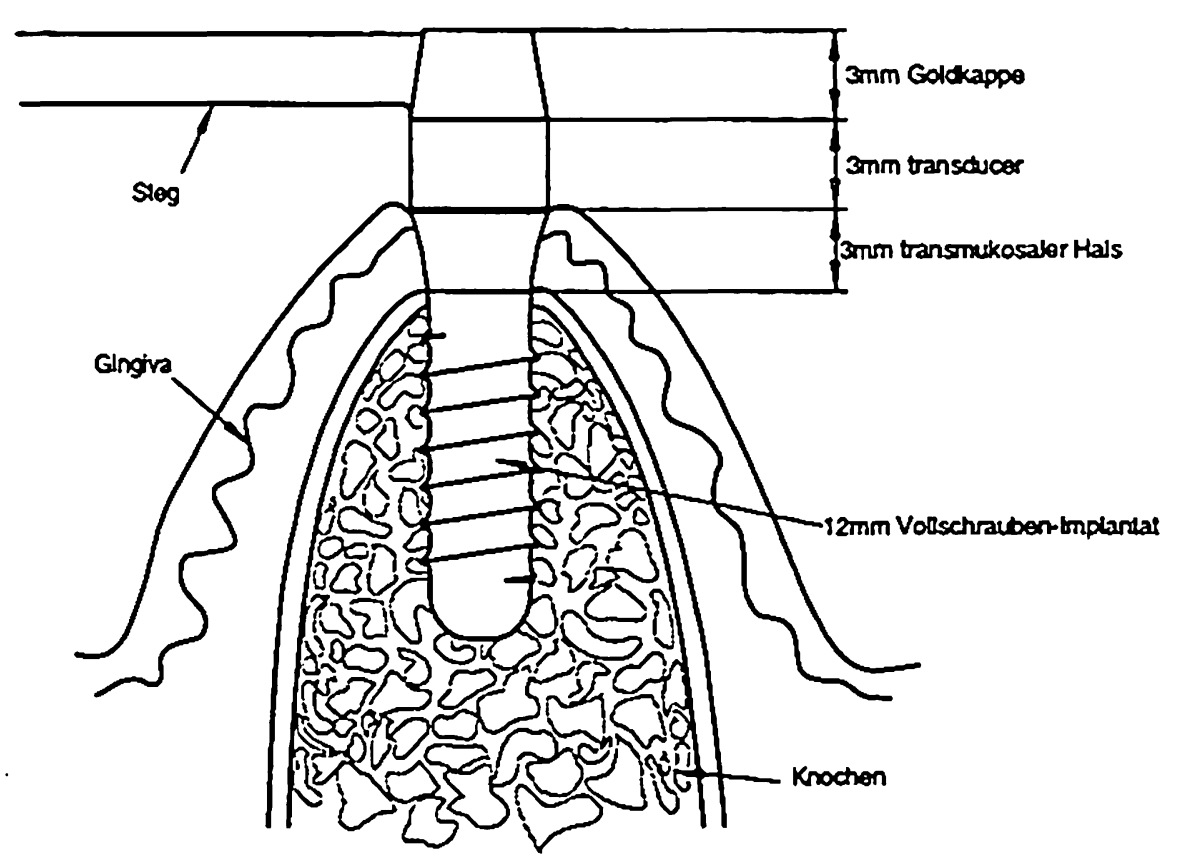

Fig. I Messanordnung, Beispiel mit Steg.

Zur Erstellung des FE Modelles wurde das Präparat eines zahnlosen Unterkiefers mit einem Somatom Plus-S (Siemens, Erlangen) Scanner und einer Schichtdicke von $1 \mathrm{~mm}$ hochauflösend vermessen. Mit Hilfe eines speziellen, validierten Interface-Programmes [2] wurde ein dreidimensionales Voxel-Modell erstellt und ins FE-Programm ANSYS 5.2 (ANSYS Inc., Houston PA) übertragen. Die Abmessungen eines einze!nen Voxels bzw. Hexaeder-Elementes betrugen dabei $1.15 \times 1.15 \times 1.0 \mathrm{~mm}$. Der E- 
Modul wurde für jedes Element mittels linearer Interpolation aus dessen mittleren HounsfieldZahl errechnet. Die Poisson-Zahl wurde einheitlich auf 0.33 gesetzt.

Innerhalb des ANSYS-Programmes wurde das Modell anschliessend um die ImplantatGeometrie ergänzt bzw. modifiziert (Fig. 2).

Die Belastung wurde in erster Linie aus den mittels Messgeber in vivo gemessenen Kräften auf den Implantaten bestimmt. Zusätzlich wurden die direkt über den Kieferkamm durch die Auflage der Prothese eingeleiteten Kräfte abgeschätzt und im Bereich der ersten Molaren ins Modell eingeleitet. Gleichzeitige Messungen mit der Bissgabel und den Kraftmessgebern zeigten, dass die absolute Kaukraft ca. um $25 \%$ über den in den Implantaten gemessenen Werten liegt.
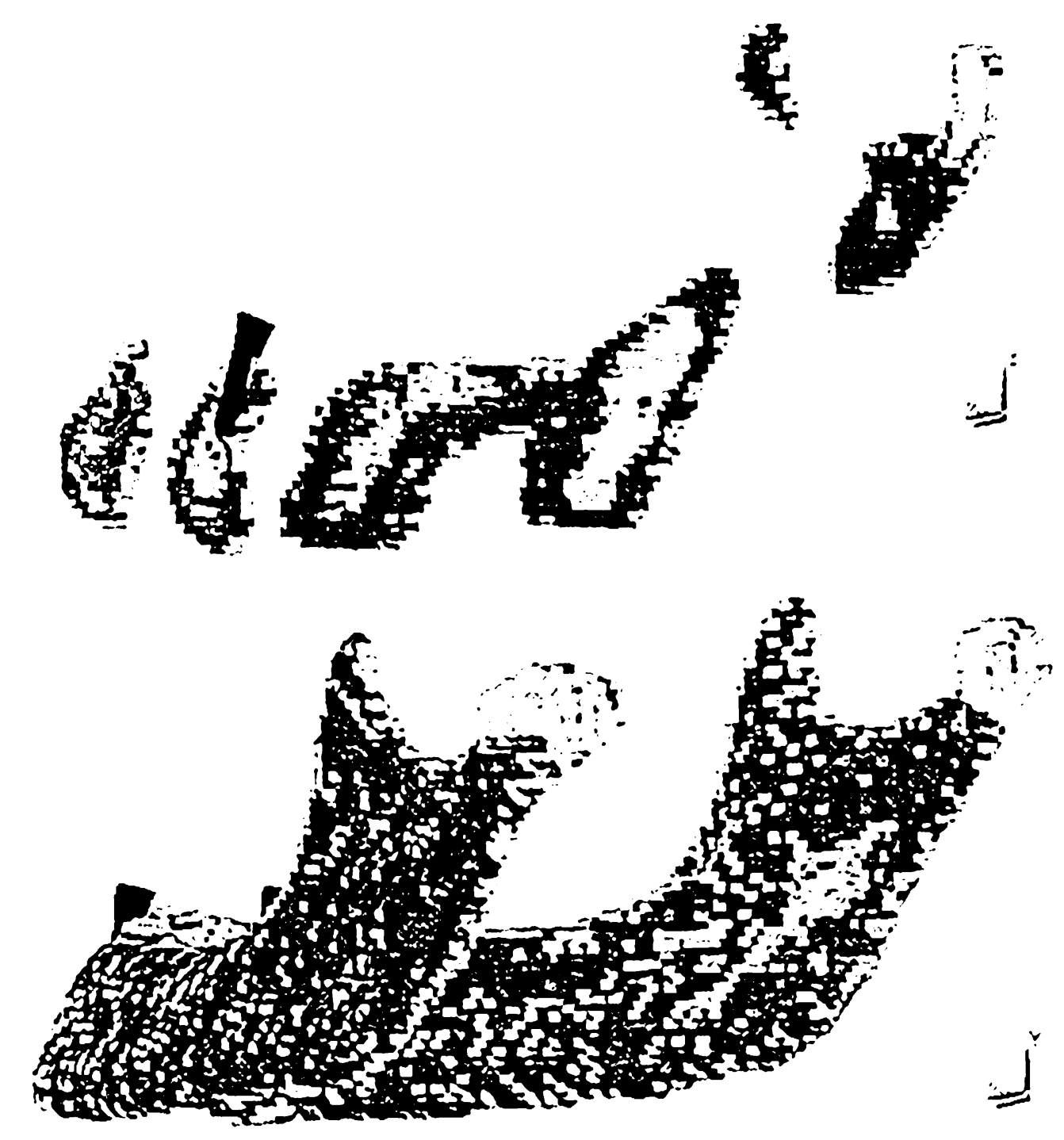

Fig. 2 Einzelne Schichten (oben) und komplettes Modell (unten). Dunklere Elemente weisen einen höhern EModul auf.

Die Einspannungen bestanden einerseits aus der Festsetzung der transversalen Freiheitsgrade in je einem Knoten in den Kondylen (Modellierung als Scharniergelenk), andererseits aus viermal je zwei Stäben, die die Masseter und Temporalis-Muskeln modellieren. Für eine erste Berechnung wurden die Belastungswerte von
Patient Nr. 5 verwendet, dessen Mandibula derjenigen des Präparates am nächsten kam. Die Belastung entsprach einer bilateralen maximalen Okklusion mit $17.1 \mathrm{~N}$ lateral gegen innen gerichtet, $32.4 \mathrm{~N}$ dorso-ventral nach vorne gerichtet und $114 \mathrm{~N}$ vertikal gegen unten gerichtet.

\section{ERGEBNISSE UND DISKUSSION}

Die dreidimensionale Kraftmessung an verschiedenen Patienten bei unterschiedlichen Aktivitäten und Implantat-Suprastrukturen zeigten, dass die nicht-vertikalen Kraftkomponenten keinesfalls vernachlässigt werden dürfen und durchaus Werte von 30-50\% der vertikalen erreichen können.

Die höchsten Implantat-Belastungen wurden im Bereich der medio-frontalen Durchtrittsstelle durch die Kortikalis gefunden, mit einer von Mises Spannung von ca. $40 \mathrm{MPa}$. Aufgrund der im Vergleich zur Implantat-Grösse relative groben Auflösung des Modelles muss dieses Resultat eher in seiner Grössenordnung bewertet werden. Für genauere Informationen wäre ein detaillierteres Submodell dieser Region nötig.

Wenn man die Belastung der gesamten Knochenstruktur betrachtet, so fällt die relativ gleichmässige Spannungs- und Dehnungsverteilung auf. Die Struktur ist offensichtlich im Sinne der funktionellen Adaptation für das Kauen und Beissen optimiert.

Mit dem vorliegenden Modell können die verschiedensten Lastfälle und Suprastrukturen bezüglich ihrer Auswirkungen durchgespielt werden, um einen globalen Überblick zu erhalten. Kombiniert mit Detailmodellen des ImplantatGrenzgebietes kann es wertvolle Hinweise auf die Belastung des Implantat-Knochen-Verbundes liefern.

\section{LITERATUR:}

[1] R. Mericske-Stern et al.; Int J Oral Maxillofac Implants 7, 185-194 (1992).

[2] J. Schmitt et al.; Biomed Technik 40: 175181 (1995). 